\title{
Memory reactivation and consolidation during sleep
}

\author{
Ken A. Paller ${ }^{1}$ and Joel L. Voss \\ Institute for Neuroscience and Department of Psychology, Northwestern University, Evanston, Illinois 60208-2710, USA
}

\begin{abstract}
Do our memories remain static during sleep, or do they change? We argue here that memory change is not only a natural result of sleep cognition, but further, that such change constitutes a fundamental characteristic of declarative memories. In general, declarative memories change due to retrieval events at various times after initial learning and due to the formation and elaboration of associations with other memories, including memories formed after the initial learning episode. We propose that declarative memories change both during waking and during sleep, and that such change contributes to enhancing binding of the distinct representational components of some memories, and thus to a gradual process of cross-cortical consolidation. As a result of this special form of consolidation, declarative memories can become more cohesive and also more thoroughly integrated with other stored information. Further benefits of this memory reprocessing can include developing complex networks of interrelated memories, aligning memories with long-term strategies and goals, and generating insights based on novel combinations of memory fragments. A variety of research findings are consistent with the hypothesis that cross-cortical consolidation can progress during sleep, although further support is needed, and we suggest some potentially fruitful research directions. Determining how processing during sleep can facilitate memory storage will be an exciting focus of research in the coming years.
\end{abstract}

The idea that memory storage is supported by events that take place in the brain while a person is sleeping is an idea that is only rarely acknowledged in the neuroscience community. At present, most memory research proceeds with no mention of any influence of sleep on memory. Nonetheless, this hypothesis is gaining empirical support. Research into connections between memory and sleep represents a burgeoning enterprise at the crossroads of traditional memory research and sleep research, an enterprise poised to provide novel insights into the human experience.

This article presents some speculations about connections between memory and sleep. We entertain the notion that declarative memories are subject to modification during sleep, and that enduring storage of such memories is systematically influenced by neural events taking place during sleep. Although other types of memory may also be subject to change during sleep (see Maquet et al. 2003), we emphasize declarative memory here.

This article also functions as an introduction to the set of papers selected for this special issue of Learning \& Memory. These papers together outline portions of the current empirical basis for memory-sleep connections, including research in humans and in nonhuman animals. The findings are tantalizing, and yet there are undoubtedly major gaps in our knowledge about the functions of sleep and about how sleep may be related to memory storage. Future research on this topic is bound to grow in exciting and unpredictable ways. Here, we explore questions about declarative memory and sleep that may serve as a useful guide for such research.

What are declarative memories and how do they change over time?

"Declarative memory" refers to the ability to recall and recognize episodes and complex facts. Our scientific understanding of declarative memory has been greatly advanced by neuropsychological analyses of patients with circumscribed amnesia. These patients have difficulty remembering episodes and facts, but are

'Corresponding author.

E-mail kap@northwestern.edu; fax (847) 467-1329.

Article and publication are at http://www.learnmem.org/cgi/doi/10.1101/ Im.75704. otherwise intellectually intact. Most notably, severe deficits in declarative memory in amnesic patients can be juxtaposed to a set of preserved memory capabilities categorized collectively as "nondeclarative memory" (see Table 1). Attempts to identify the fundamental differences between declarative and nondeclarative memory have been useful for elucidating the unique neural and cognitive characteristics of declarative memory.

Many memory theorists concur that declarative memories are distinctive in their dependence on multiple neocortical regions (e.g., Squire and Butters 1984, 1992; Squire 1987; Schacter and Tulving 1994; Schacter 1996; Mayes and Downes 1997; Eichenbaum and Cohen 2001; Squire and Schacter 2002). For example, someone's memory for a symposium on memory and sleep held in Chicago on a Saturday in 2003 might include the spatial layout of the meeting room, the visual features of the environment and of the people in the room, the Chicago skyline visible from the windows, images of the speakers making their presentations, auditory characteristics of the speaker's voices, the sequence of events in the symposium, the content of the presentations, emotional coloring of these events, other events that led up to the symposium, events just after the symposium, and episodes from subsequent days when the symposium was remembered and discussed with other people. These varied features would be represented in multiple cortical regions that are specialized for processing different sorts of information. In this example, and in general, declarative memory depends fundamentally on representations that include conjunctions among various elements. In other words, the memory can be said to require "cross-cortical storage," in that the fragments by themselves are not sufficient to comprise the memory, but rather, these fragments must be linked together as a cohesive unit for the memory to exist (Paller 1997, 2002). The corresponding cognitive characteristics of declarative memory probably include a reliance on relational representations (Eichenbaum and Cohen 2001; Shimamura 2002). Precisely specifying the relationship between cross-cortical storage and relational representations remains a top goal for future memory research.

Here, we conceptualize declarative memory not only by emphasizing cross-cortical storage, but further, we postulate that this neural feature may be the essential characteristic of declara- 
Table 1. Contrasting declarative memory with other types of memory

\begin{tabular}{|c|c|c|}
\hline Declarative memory & Examples & Findings in patients with amnesia \\
\hline Declarative memory & $\begin{array}{l}\text { Recall and recognition of episodes and } \\
\text { facts (i.e., episodic memory and } \\
\text { semantic memory) }\end{array}$ & $\begin{array}{l}\text { Impairment in storage, producing deficits } \\
\text { in new learning (anterograde amnesia) } \\
\text { and in remembering information } \\
\text { acquired prior to the illness or injury } \\
\text { (retrograde amnesia) }\end{array}$ \\
\hline Immediate memory & $\begin{array}{l}\text { Information kept in mind by continuous } \\
\text { rehearsal (e.g., verbal working memory) }\end{array}$ & Preserved \\
\hline Nondeclarative memory: & & $\begin{array}{l}\text { Generally preserved, but with some } \\
\text { notable exceptions }\end{array}$ \\
\hline Perceptual priming & $\begin{array}{l}\text { Speeded or more accurate responses to a } \\
\text { stimulus, based on perceptual factors }\end{array}$ & $\begin{array}{l}\text { Preserved if performance is not } \\
\text { contaminated by declarative memory } \\
\text { (i.e., using an implicit memory test that } \\
\text { discourages explicit memory retrieval) }\end{array}$ \\
\hline Conceptual priming & $\begin{array}{l}\text { Speeded or more accurate responses to a } \\
\text { stimulus, based on conceptual factors }\end{array}$ & $\begin{array}{l}\text { Preserved in some cases, but further } \\
\text { investigation is required, particularly } \\
\text { across stimulus domains }\end{array}$ \\
\hline Skills & $\begin{array}{l}\text { Behaviors that improve gradually with } \\
\text { practice, including cognitive skills (e.g., } \\
\text { reading mirror-reversed text) and motor } \\
\text { skills }\end{array}$ & $\begin{array}{l}\text { Preserved when skill acquisition is } \\
\text { accomplished without reliance on } \\
\text { declarative memory (which is often not } \\
\text { the case for skills learned outside the } \\
\text { laboratory) }\end{array}$ \\
\hline Classical conditioning & $\begin{array}{l}\text { Learned associations between two stimuli, } \\
\text { one of which elicits an automatic } \\
\text { response }\end{array}$ & $\begin{array}{l}\text { Preserved under conditions with temporal } \\
\text { overlap between conditioned and } \\
\text { unconditioned stimuli }\end{array}$ \\
\hline
\end{tabular}

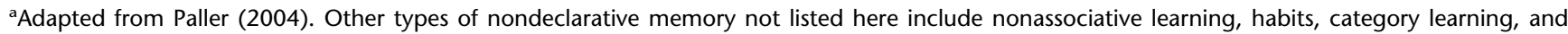
artificial grammar learning.

tive memory that distinguishes it from nondeclarative memory. Subtypes of nondeclarative memory such as motor skills, cognitive skills, and conditioning may also involve plasticity and consolidation, but storage depends on specialized networks in the brain other than cross-cortical links.

An intriguing ramification of this concept of cross-cortical storage is that the complex nature of these memory representations may be a necessary prerequisite for the conscious experience of remembering, "conscious recollection." Remembering the multidimensional features of an episode, particularly spatiotemporal contextual features, can lead naturally to the inference that one is bringing to mind a memory from one's past, hence, recollection. However, cross-cortical storage is not sufficient for recollection. The conscious experience of remembering declarative memories also depends on working memory, attention, cognitive control, and metamemory-functions supported in part by prefrontal cortex.

A particular feature of cross-cortical storage that is highly relevant for present purposes is that the memory representations in the cerebral cortex are formed gradually (McClelland et al. 1995; Squire and Alvarez 1995; McClelland 1998; O'Reilly and Norman 2002). The hippocampus is thought to play the special role of quickly allowing connections between separate cortical representations. These quick links are temporary-cross-cortical linkage is ultimately mediated by cortico-cortical connections and by new "coherence ensembles" (Paller 1997) that link distinct cortical representations. In any event, declarative memories exist in a fragile state after initial learning. Enduring declarative memories are those that have undergone a sufficient amount of cross-cortical consolidation, such that hippocampal neurons are no longer required to unitize the set of distinct cortical networks.

A key facet of declarative memory is thus that individual memories undergo change after initial learning. Findings from studies of retrograde amnesia have provided indications concerning the length of time required for cross-cortical consolidation to produce a cortically self-sufficient memory. However, we suggest that the time-frame of this process is not uniform, but rather that it depends on the extent to which a memory is retrieved and associated with other stored information. Cross-cortical consolidation is an active process that depends on the nature and frequency of memory access during the time period from initial acquisition to retrieval. When declarative memory access occurs, the cortical fragments of the episodic or factual representation can become more thoroughly bound together, more strongly linked to new representations of holistic aspects of the episode or fact, and also associated with related stored information. Connections to related information enrich the memory in question and provide additional routes for subsequent retrieval. The related stored information can include information that was acquired prior to the new learning as well as information acquired subsequently.

We can now summarize the logic of our position as follows:

1. Declarative memories change as a result of postacquisition processing. This processing can promote cross-cortical consolidation and generate memories that can be retrieved in the absence of an intact hippocampus.

2. Portions of declarative memories are accessed during sleepreports of dream content frequently show that fragments of recent events as well as other bits of knowledge are incorporated into sleep mentation.

3. Accordingly, processing during sleep can influence the course of cross-cortical consolidation and promote the transformation of declarative memories. The time course of this transformation is not limited. Consolidation can begin immediately after an episode occurs and it can progress further whenever the declarative memory is modified in relation to other information, including information acquired many years later.

Our proposal overlaps with those presented by others, but also differs in important respects. Our hypothesis differs from the "relabilization" idea of Walker et al. (2003), in that we propose that memory access is directly associated with consolidation, not that memory access during sleep can push memories into a modifiable or destabilized state. Our view also differs from that of 
Ribeiro and Nicolelis (2004), in that we stress connections among dispersed neocortical networks as well as hippocampalneocortical connections, and not a movement of information from hippocampus to neocortex. Likewise, our proposal differs from the "unlearning" hypothesis of Crick and Mitchison (1983), in that we emphasize the formation and strengthening of associations, not their active removal. Payne and Nadel (2004) also emphasize memory improvement due to sleep, and they suggest that dream events can be relatively coherent when corticohippocampal networks are operative early in the night, or fragmented and bizarre when cortical networks are engaged in isolation later in the night. They further speculate that high levels of cortisol disrupt cortico-hippocampal connectivity late in the night, particularly during REM ${ }^{2}$ periods (Buzsáki 1996), and that episodic memory consolidation proceeds more effectively earlier in the night when REM periods are less frequent. Gais and Born (2004) emphasize the beneficial effects of slow-wave sleep for declarative memories, with special reference to EEG coherence signals that may index this processing. Remarkably, transcranial direct current stimulation can apparently facilitate this memory processing. However, Gais and Born (2004) still describe a transfer of information from hippocampal to neocortical networks, contrary to the cross-cortical consolidation that we suggest takes place in concert with the formation of new coherence ensembles. We have not surveyed all other theories that impinge on sleep/ memory connections, but we refer the interested reader to several recent summaries (Maquet 2001; Stickgold et al. 2001; Hobson and Pace-Schott 2002; Maquet et al. 2003; Rock 2004) and to the other articles in this special issue of Learning \& Memory.

\section{Does off-line processing of declarative memories take place during sleep?}

Jonathan Winson developed a detailed and provocative theory concerning memory and sleep (Winson 1985, 2002, 2004). According to Winson, dreams are not simply a peculiar consequence of random brain activity, but rather reflect meaningful memory processing. In sharp contrast with the Freudian view that dream content is determined by unconscious drives disguised and filtered due to repression, Winson proposed that dreams are part of an adaptively advantageous mechanism for evaluating recent experiences and relating them to behavioral strategies and goals. Sleep mentation can thus result in better memory and expanded perspectives on our daily problems and concerns, as memories of recent and not-so-recent experiences are accessed, new connections between experiences are forged, and cross-cortical consolidation moves forward.

This processing of memories during sleep may be an effective way to organize memories so that they will subsequently be available when needed. Winson further hypothesized that an alternative approach to achieve the same end would be to organize memories during the waking state, and that this would require a disproportionately large prefrontal cortex. Memory processing during sleep was postulated to be an invention of early mammals that developed into today's placental and marsupial mammals. These species show clear evidence of sleep stages including REM and slow-wave sleep. On the other hand, novel polysomnographic results have been obtained in monotremes,

${ }^{2}$ REM sleep is a stage of sleep characterized by abundant rapid eye movements, high-frequency EEG signals similar to those during alert waking periods, and other polysomnographic indicators. Dream reports are often elicited upon awakening from REM sleep, but dreams and other sorts of sleep mentation also occur during non-REM sleep. The deepest state of non-REM sleep is known as slow-wave sleep and is characterized by low-frequency $(0.5-2 \mathrm{~Hz})$ EEG oscillations. A progression of sleep stages generally occurs in cycles roughly $90 \mathrm{~min}$ in length, with progressively longer REM periods over the course of a night's sleep. the order of egg-laying mammals that consists of duck-billed platypus and spiny anteater and that probably typifies a very early stage in mammalian evolution. Monotremes do not appear to have the same complexity of sleep stages $^{3}$ (Allison et al. 1972; Siegel et al. 1999) and also possess frontal lobes that comprise a disproportionately large proportion of the cerebral cortex relative to that in other mammals. Winson suggested that monotremes use their large prefrontal cortex for memory reprocessing during waking, whereas all other mammals accomplish this function more efficiently during sleep.

A variety of physiological observations may be relevant for understanding memory processing during sleep. Physiological studies in animals are described in two articles in this special issue (Battaglia et al. 2004; Ribeiro and Nicolelis 2004). Hippocampal theta rhythm is a phenomenon observed in animals during waking experiences that are generally important for members of that particular species to remember (Winson 1985). Hippocampal theta also occurs during REM sleep in these animals, when the information acquired during waking may undergo additional processing, perhaps related to memory formation (Buzsáki 2002). Synchronous theta in multiple medial temporal regions can influence long-term potentiation (Lynch et al. 1990), and thereby may dictate modulation of neocorticalhippocampal interconnections.

Evidence from human subjects also points to possible connections between theta activity and memory (Klimesch et al. 2001; Fell et al. 2002; Sederberg et al. 2003). This evidence has been obtained from EEG recordings made with electrodes on the scalp and with electrodes implanted in neocortical and hippocampal regions. Two recent studies highlight the possibility of establishing closer connections between human hippocampal activity and sleep, based on recordings from intracranial electrodes. In single-cell recordings in humans, bursts of hippocampal neuronal activity have been observed during slow-wave sleep (Staba et al. 2002). In another study, short bursts of hippocampal theta activity were observed during REM sleep (Cantero et al. 2003). Although these oscillations were apparently not synchronized with neocortical theta activity, recordings from rodents have revealed temporal coupling of EEG oscillations during slow-wave sleep, indicative of communication between neocortical and hippocampal cell assemblies (Sirota et al. 2003).

In recordings of single neuron activity during sleep in rats, place cells in the CA1 field of the hippocampus show altered firing based on waking experience, and populations of such cells show patterns of activity that mirror spatial coding patterns recently exhibited in new environments in the waking state (Pavlides and Winson 1989; Wilson and McNaughton 1994; Battaglia et al. 2004). Also, sleep systematically induces the expression of genes that have been implicated in supporting synaptic plasticity during wakefulness (Ribeiro and Nicolelis 2004). Gating of information through the trisynaptic circuit of the hippocampus changes systematically during sleep stages (e.g., Winson and Abzug 1977), perhaps regulating memory access so as to promote the development of new connections between hippocampal networks and neocortical networks.

\section{Why might memory processing during sleep be useful in humans?}

Analyses of dream reports include a wealth of perspectives on the meaning of dreams, and a variety of opinions have been ex-

\footnotetext{
${ }^{3}$ In both the spiny anteater (echidna) and the platypus, it is likely that REM sleep is absent altogether (T. Allison, pers. comm., July 2004), whereas slowwave sleep is present, which has been taken to imply that slow-wave sleep evolved much earlier in mammalian evolution than did REM sleep (Allison et al. 1972).
} 
pressed about why or whether dreaming is useful. By eliciting multiple reports on the same night, Rosalind Cartwright discovered that consecutive dreams can sometimes all relate to the same common theme (Cartwright 1990; see also Cartwright 2004). For example, recently divorced individuals produced dreams that appeared to be systematically related to their strategies for coping with some current challenge. Generally, earlier dreams in the night include memory fragments from recent experiences, whereas later dreams incorporate memory fragments from increasingly farther back in the past (e.g., Roffwarg et al. 1978; see also Walker et al. 2003). Thus, an intriguing possibility, as noted by Winson (1985), is that dreaming exposes a mechanism whereby emotional issues can be worked through and behavioral strategies can be developed and adjusted with reference to experiences from the preceding days as well as older experiences. By this scenario, there is no special need for dream content to be remembered. Two benefits of sleep cognition result whether or not dreams are recalled upon waking (1) tuning of behavioral strategies, and (2) reorganization of memories.

To the extent that this conception of sleep cognition is accurate, there are several implications for theories of declarative memory. First, memories may not lie dormant during sleep, but rather may be receiving regular exercise. Second, cross-cortical consolidation may occur on a systematic basis due to memory access during sleep. The timing and the frequency of memory access during sleep would be guided by the relevance of each memory to current issues affecting an individual's sleep cognition and the problem-solving strategies applied to these issues. The time course of cross-cortical consolidation would be determined accordingly, such that memories processed intensively in this manner would more readily become cortically self-sufficient.

Given the premise that our brains cannot preserve all memories for all details for all of our experiences, some heuristics would be helpful for determining which declarative memories should be maintained. A useful strategy might be to place emphasis on those memories that are relevant to personally important issues currently under active consideration. In this way, cross-cortical consolidation would progress as a function of the extent to which a memory is actively processed in relation to other stored information. The speed with which a new memory is transformed into an enduring memory would be based on this problem-oriented processing rather than on a random or inexorable biochemical process. Memory storage would rapidly reach an asymptotic state when a declarative memory became thoroughly integrated with frequently accessed information and with one's behavioral strategies and personality.

\section{How should dream reports be interpreted?}

Dream interpretation is notoriously tricky-sometimes a cigar is just a cigar. Nevertheless, dream reports have provided firm evidence that our experiences during the day are regularly incorporated into our dreams. Although a dream may appear to have meaning when it is recounted, an in-depth analysis of the dream report is not likely to accurately reveal the mechanism whereby the dream was actually produced. Here, we take the position that dreams are not necessarily distorted reflections of drives and wishes or some secret information waiting to be revealed. Indeed, in some cases dream content may give a misleading impression about the functions of sleep cognition.

Whereas the grand function or functions of sleep remain to be elucidated, we speculate that one adaptive function accomplished during sleep involves assembling an individual's recent experiences of the past few days along with their goals, desires, and problems. Bringing this information together during sleep would allow stored information to be accessed and consolidated in a systematic and highly advantageous manner; memories processed in this way would tend to be those that will be useful in subsequent waking experiences.

The narrative created on the basis of the fragmentary information activated during sleep, however, may be tangential to the memory function actually accomplished. Dream content may merely reflect a story spontaneously produced to connect the fragments. The story itself may obscure the fact that memory access results in the formation of new connections between memories, including both recent and older memories. These discrete connections between memories may be central for both consolidation and problem solving. In other words, when we interpret dream content and manufacture meaning that goes well beyond the individual features of a dream, we are in danger of missing the trees by attributing too much significance to the forest. The principal benefit of dreaming may be the new connections formed between pairs of memory fragments. Our storehouse of declarative memories may be enriched by the establishment of new connections between a fragment of one episode and a fragment of another episode. These new connections could act to expand the relevance of a particular episode and increase the meaningfulness of individual features. Futhermore, new fragment-to-fragment connections could also function on a larger scale of connectivity as new relationships are forged between features of these episodes and general behavioral strategies. Behavioral strategies are, in a sense, also cognitive fragments based on past experiences, but they become enduring aspects of one's personality and dictate how we respond to subsequent events. In this way, strategies and goals can come to influence the course of consolidation.

The possibility that the fundamental building blocks of dreams are memory fragments rather than coherent episodes is consistent with evidence from dream reports. For example, dreams appear to include small pieces of recent episodes rather than entire autobiographical events (Fosse et al. 2003). Experiences available upon awakening may be composed of cortical fragments of declarative memories rather than entire episodes that include hippocampal links among cortical fragments. In this case, the assumption that the neural events engaged during dreaming are faithfully made evident in the structure of the dream narrative could lead to the inference that memory consolidation during sleep is strictly nonassociative and does not involve the hippocampus. This position conflicts with the notion that sleep promotes declarative memory processing and the formation of new relationships between stored information. Given this discrepancy, we prefer to assume that the information content that reaches awareness in the form of remembered dreams does not provide a straightforward glimpse into the memory processing accomplished during sleep. Evidence from other sources will be needed to determine how memory processing during sleep contributes to the cross-cortical consolidation of declarative memories.

A particularly intriguing clue about memory and dreaming, given the present emphasis on memory for episodes, is that in the typical transition from dreaming to waking, the content of a dream is experienced in an extraordinarily fleeting manner. Often, a dream can only be remembered to the extent that it is rehearsed upon awakening. At the precise time of awakening, the dream apparently exists in a state quite different from that of normal waking experiences, which are readily available to be stored. The dreamer must immediately rehearse the dream in order for it to be transformed into the active state of perceptual experiences. Only after this transformation in the representational form is the dream available for lasting storage as a declarative memory. 
Why don't dreams automatically emerge fully formed in the episodic buffer of working memory upon awakening? This peculiar aspect of dreaming may result from an abrupt change in hippocampal gating at the moment of awakening. Altered hippocampal gating during sleep stages may reflect alterations in how information flows between neocortical networks and hippocampal networks (Winson 1985; Buzsáki 1996). Given this gating of information, consolidation may progress, not because of a single sleep stage, but by virtue of some complex interplay among complementary mechanisms over the course of a good night's sleep-like a progression of distinct movements that together constitute a symphony. For example, one possibility is that hippocampal-cortical interactions are restricted during REM sleep, but that hippocampal activity during REM sleep nonetheless sets the stage for memory processing, influencing cortical rewiring that happens subsequently, perhaps during periods of slow-wave sleep.

Dream interpretation may still provide some insight into mental activity that otherwise might remain unconscious, even given that dreams are not a product of Freudian repression, unconscious drives, and wish fulfillment. Instead, consider that nondeclarative memory includes a vast set of dispositions and conditioned behavioral patterns that, by their nature, are not accessible to conscious recollection. Understanding these influences on our behavior can be advantageous. Achieving this goal in psychoanalysis and psychotherapy may be aided by dream analysis, not by reviving forgotten declarative memories or converting unconscious memories to conscious ones, but by understanding nondeclarative memory-the habits and behavioral predilections that guide so much of our behavior.

\section{Sleep and declarative memory: Where do we go from here?}

Whereas a steadily increasing amount of evidence indicates that memory consolidation occurs during sleep, a great deal of uncertainty remains about which specific elements of memories are reprocessed and which portions of the complex architecture of sleep are relevant. Firmly establishing specific sleep-memory connections will require a blending of the methods of traditional sleep and memory research and may be the best way to convincingly determine the fundamental functions of sleep. Furthermore, these efforts may transform the investigation of memory by providing a better understanding of how declarative memories change over time.

Many empirical approaches have demonstrated that sleep can influence subsequent waking cognition (e.g., Kuriyama et al. 2004; Smith et al. 2004). Navigating these approaches requires avoiding some tricky methodological potholes. Sleep deprivation, for example, can lead to nonspecific effects, such as stress induction and lowered arousal levels, and disrupting one sleep stage can alter subsequent sleep stages. It is thus not surprising that the literature in this area is mixed, but despite these limitations, some convincing evidence has been obtained. One recent example is a study examining effects of sleep on the development of insight (Wagner et al. 2004). Subjects were required to perform mathematical operations on a string of digits. The time taken to complete this task could be drastically reduced if a "hidden rule" was suddenly discovered. This insight was more frequent when training on this task was followed by a night's sleep than by either a sleepless night or by an equivalent duration of daytime wakefulness. The intervening period of sleep that seemed to facilitate insight might reflect some reprocessing of memory during sleep, as links are created between pieces of information that otherwise might remain disparate. Even though the mathematical task was not a task of declarative memory, the findings fit with our description of declarative memory processing during sleep. Future work in this area is needed to build on such findings by clarifying the neurocognitive events during sleep that can influence declarative memory storage.

Although sleep can be categorized into stages that exhibit unique microstructures, this classic understanding of the structure of sleep may be deceiving in its apparent simplicity. Sleep has an incredibly complex and interdependent architecture. Judging by the stereotyped biological events that occur during distinct phases of sleep (Hellman and Abel 2003; Jones 2004; Steriade 2004), the information processing transactions in one phase of sleep depend on the integrity of prior sleep periods and can influence future periods. Notably, the spatiotemporal firing sequence of hippocampal neurons during REM sleep can mimic that seen during a prior learning phase (Battaglia et al. 2004). This "replay" presumably serves to strengthen synaptic connections between neurons that process information in a manner relevant to performing the task-replay may engage mechanisms for circuitry remodeling produced by long-term potentiation. The success of this modification would be highly dependent on catecholaminergic/indolaminergic neuromodulation (Kilgard and Merzenich 1998; Bao et al. 2001) that is known to occur during the period of slow-wave sleep prior to the REM period of replay (Hellman and Abel 2003).

This interdependence of sleep stages makes the interpretation of sleep deprivation studies particularly difficult. Deficits in behavior following deprivation cannot readily be ascribed to the deprived stage per se. This potentially nonspecific aspect of the sleep deprivation methodology must be taken into account when designing new experiments to explore the function of particular sleep stages. Deprivation studies must be re-examined in this light and alternative interpretations considered. For example, the apparent immunity of declarative memories to REM sleep disruption (Stickgold 2003) might be due to compensatory REM-like consolidation during the hypnagogic period that occurs when falling back to sleep following the disruption.

Given sleep-stage interdependence, it follows that memory processing during sleep may depend on the confluence of different processing steps occurring in different sleep stages. Taking this idea one step further, processing during waking and during sleep may play complementary roles in memory consolidation. We have insufficient evidence to know whether cross-cortical consolidation during sleep is the same or different from crosscortical consolidation during waking, but this is an interesting topic for future investigation.

An additional factor that complicates the task of determining the exact role of specific types of sleep in the consolidation of declarative memories is that declarative memories are themselves not unitary phenomena. Declarative memories are built by the interaction of different neural networks supporting different components of memory. These components generally include, at the very least, memory for the individual items comprising an event plus associative links among these items (Eichenbaum and Cohen 2001; Davachi et al. 2003), the multidimensional context in which learning occurs (Cabeza et al. 2002; Takahashi et al. 2002), and emotional experiences unleashed by an event (Canli et al. 2000).

A promising approach for sleep/memory research may be to explore possible connections between particular sleep signals and certain aspects of declarative memories. For example, it may be possible to monitor learning that takes place over the course of an uninterrupted night's sleep by focusing on only a single facet of declarative memory. Learning associations among a set of items presented together in a scene might be facilitated to the extent that those scenes are replayed during sleep. Perhaps performance gains would be associated with alterations in indices of

\section{Learning \& Memory}


sleep microstructure (e.g., EEG signals such as theta-band coherence, PGO spikes, K-complexes, and so on). Certain EEG signals may provide markers for memory processing during sleep. Remarkably, findings using transcranial direct current stimulation suggest that slow oscillatory activity during slow wave sleep can be modulated so as to benefit declarative memory (Gais and Born 2004; Marshall et al. 2004). A recent study in rats used a related strategy in investigating pontine waves (Datta et al. 2004). When pontine waves were induced during sleep via carbachol injection to the subcoeruleus nucleus, the deleterious effects of REM sleep deprivation on learning in a shuttle-box avoidance paradigm were precluded. Given the role of the subcoeruleus nucleus in the generation of pontine waves and the monosynaptic projections from subcoeruleus nucleus to hippocampus, this particular element of sleep microstructure may be closely related to the success of memory processing during REM sleep. In general, correlating unitary declarative memory phenomena with individual elements of sleep microstructure might identify specific neurophysiological events that are relevant to declarative memory processing during sleep. Furthermore, sleep analyses in patient populations may provide clues to disorders of memory.

The conceptualization of declarative memory outlined above emphasized that an enduring declarative memory depends on a process of cross-cortical consolidation that generally results from multiple retrieval events over an extended period of time. One of the difficulties in observing these progressive changes in memory storage is the uncertainty of memory access. A detailed elucidation of the neuroanatomical, neurophysiological, neurochemical, and functional properties of the component processes of sleep could provide a unique window into memory consolidation. Functional brain imaging, for example, may help localize neural processes that lead to improved performance, such as in perceptual discrimination (Schwartz et al. 2002). Although there are many practical problems to surmount, neuroimaging methods may eventually allow us to monitor the neural activity that leads to the consolidation of distinct aspects of declarative memories.

A wealth of exciting new findings can be expected from future research aimed at uncovering connections between sleep and declarative memory. Success will require an alliance between the traditionally separate endeavors of memory research and sleep research. Contemporary hypotheses about sleep-memory connections will be transformed considerably in the process.

\section{Conclusions}

Here, we have sketched a tentative proposal about sleep and declarative memory-that sleep is essentially a nightly session of psychotherapy. The ultimate challenge of declarative memory is how to use the massive influx of new information gained each day to best advantage. One's ever-expanding storehouse of declarative memories requires continual restructuring as new information is added. Perhaps sleep provides a vehicle whereby this memory reactivation and consolidation can be moved forward while taking current difficulties facing the individual into account. One's behavioral strategies for dealing with daily life may thus exert an influence on how memory storage proceeds-and be influenced, in turn, by the memory change that occurs. Crosscortical consolidation proceeds so that some information comes to be represented more strongly, with richer connections to other stored information. Other information is seldom accessed and does not benefit from cross-cortical consolidation. Some memories are subject to distortion, some are subject to massive interference, and some are lost forever. Memory change is inexorable, but the many hours we spend sleeping may actually serve to boost the usefulness of memory change.

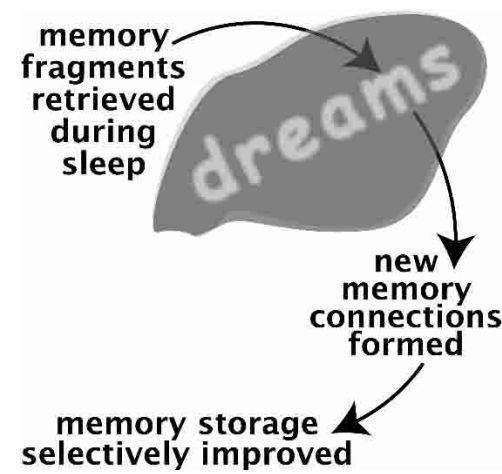

Figure 1. Schematic representation of memory reactivation and consolidation during sleep.

When we are awake, we have the opportunity to learn, to acquire new information, to relate new information to what we already know, and to creatively put information together in novel ways. When we are asleep, memory storage may progress similarly. This idea is shown schematically in Figure 1. Although memory processing during sleep is generally not under volitional $\mathrm{control}^{4}$, it may nonetheless be structured so as to help satisfy an individual's current needs and goals.

\section{Acknowledgments}

We thank the contributors to this special issue, most of whom also participated in the symposium on sleep and memory held in October of 2003 in Chicago. We are especially indebted to Jonathan Winson for his inspirational ideas on these issues, which we have hopefully conveyed accurately here. Research support from Northwestern University and from NINDS grant NS34639 is gratefully acknowledged.

\section{References}

Allison, T., Van Twyver, H., and Goff, W.R. 1972. Electrophysiological studies of the echidna, Tachyglossus aculeatus. I. Waking and sleep. Arch. Ital. Biol. 110: $145-184$.

Bao, S., Chan, V.T., and Merzenich, M.M. 2001. Cortical remodelling induced by activity of ventral tegmental dopamine neurons. Nature 412: 79-83.

Battaglia, F.P., Sutherland, G.R., and McNaughton, B.L. 2004 Hippocampal sharp wave bursts coincide with neocortical "up-state" transitions. Learn. Mem. (this issue).

Buzsáki, G. 1996. The hippocampo-neocortical dialogue. Cerebral Cortex 6: 81-92.

. 2002. Theta oscillations in the hippocampus. Neuron 33: $325-340$.

Cabeza, R., Anderson, N.D., Locantore, J.K., and McIntosh, A.R. 2002. Aging gracefully: Compensatory brain activity in high-performing older adults. Neuroimage 17: 1394-1402.

Canli, T., Zhao, Z., Brewer, J., Gabrieli, J.D., and Cahill, L. 2000. Event-related activation in the human amygdala associates with later memory for individual emotional experience. J. Neurosci. 20: RC99.

Cantero, J.L., Atienza, M., Stickgold, R., Kahana, M.J., Madsen, J.R., and Kocsis, B. 2003. Sleep-dependent theta oscillations in the human hippocampus and neocortex. J. Neurosci. 23: 10897-10903.

Cartwright, R.D. 1990. A network model of dreams. In Sleep and cognition (eds. R.R. Bootzin et al.), pp. 179-189. American Psychological Association, Washington, D.C.

. 2004. The role of sleep in changing our minds: A psychologist's discussion of papers on memory reactivation and consolidation in sleep. Learn. Mem. (this issue).

Cicogna, P. and Bosinelli, M. 2001. Consciousness during dreams. Consciousness and Conition 10: 26-41.

${ }^{4}$ We set aside the special circumstances of "lucid dreaming," when an individual becomes aware that a dream is unfolding and may orchestrate subsequent dream content (LaBerge 1990; Varela 1997; Cicogna and Bosinelli 2001). Future investigations are needed to explore possible ramifications of controlling one's dreams on memory consolidation. 
Crick, F. and Mitchison, G. 1983. The function of dream sleep. Nature 304: 111-114.

Datta, S., Mavanji, V., Ulloor, J., and Patterson, E.H. 2004. Activation of phasic pontine-wave generator prevents rapid eye movement sleep deprivation-induced learning impairment in the rat: A mechanism for sleep-dependent plasticity. J. Neurosci. 24: 1416-1427.

Davachi, L., Mitchell, J.P., and Wagner, A.D. 2003. Multiple routes to memory: Distinct medial temporal lobe processes build item and source memories. Proc. Natl. Acad. Sci. 100: 2157-2162.

Eichenbaum, H. and Cohen, N.J. 2001. From conditioning to conscious recollection: Memory systems of the brain. Oxford University Press, New York.

Fell, J., Klaver, P., Elger, C.E., and Fernandez, G. 2002. The interaction of rhinal cortex and hippocampus in human declarative memory formation. Rev. Neurosci. 13: 299-312.

Fosse, M.J., Fosse, R., Hobson, J.A., and Stickgold, R.J. 2003. Dreaming and episodic memory: A functional dissociation? J. Cogn. Neurosci. 15: 1-9.

Gais, S. and Born, J. 2004. Declarative memory consolidation: Mechanisms acting during human sleep. Learn. Mem. (this issue).

Hellman, K. and Abel, T. 2003. Molecular mechanisms of memory consolidation in sleep and brain plasticity. In Sleep and brain plasticity (eds. P. Maquet et al.), pp. 295-325. Oxford University Press, New York.

Hobson, J.A. and Pace-Schott, E.F. 2002. The cognitive neuroscience of sleep: Neuronal systems, consciousness and learning. Nat. Rev. Neurosci. 3: 679-693.

Jones, B.E. 2004. Activity, modulation and role of basal forebrain cholinergic neurons innervating the cerebral cortex. Prog. Brain Res. 145: $157-169$

Kilgard, M.P. and Merzenich, M.M. 1998. Cortical map reorganization enabled by nucleus basalis activity. Science 279: 1714-1718.

Klimesch, W., Doppelmayr, M., Yonelinas, A., Kroll, N.E., Lazzara, M., Rohm, D., and Gruber, W. 2001. Theta synchronization during episodic retrieval: Neural correlates of conscious awareness. Cogn. Brain Res. 12: $33-38$.

Kuriyama, K., Stickgold, R., and Walker, M.P. 2004. Sleep-dependent learning and motor skill complexity. Learn. Mem. (this issue).

LaBerge, S. 1990. Lucid dreaming: Psychophysiological studies of consciousness during REM sleep. In Sleep and cognition (eds. R.R. Bootzin et al.), pp. 109-126. American Psychological Association, Washington, D.C.

Lynch, G., Kessler, M., Arai, A., and Larson, J. 1990. The nature and causes of hippocampal long-term potentiation. Prog. Brain Res. 83: $233-250$.

Maquet, P. 2001. The role of sleep in learning and memory. Science 294: $1048-1052$

Maquet, P., Smith, C., and Stickgold, R. 2003. Sleep and brain plasticity. Oxford University Press, New York.

Marshall, L., Mölle, M., Hallschmid, M., and Born, J. 2004. Transcranial direct current stimulation (tDCS) during sleep improves memory. $J$. Neurosci (in press).

Mayes, A.R. and Downes, J.J. 1997. Theories of organic amnesia. Psychology Press, Hove, East Sussex, UK.

McClelland, J.L. 1998. Complementary learning systems in the brain. A connectionist approach to explicit and implicit cognition and memory. Ann. N.Y. Acad. Sci. 843: 153-169.

McClelland, J.L., McNaughton, B.L., and O'Reilly, R.C. 1995. Why there are complementary learning systems in the hippocampus and neocortex: Insights from the successes and failures of connectionist models of learning and memory. Psychol. Rev. 102: 419-457.

O'Reilly, R.C. and Norman, K.A. 2002. Hippocampal and neocortical contributions to memory: Advances in the complementary learning systems framework. Trends Cogn. Sci. 6: 505-510.

Paller, K.A. 1997. Consolidating dispersed neocortical memories: The missing link in amnesia. Memory 5: 73-88.

. 2002. Cross-cortical consolidation as the core defect in amnesia: Prospects for hypothesis-testing with neuropsychology and neuroimaging. In Neuropsychology of memory (eds. L.R. Squire and D.L. Schacter), pp. 73-87. Guilford Press, New York.

. 2004. Electrical signals of memory and of the awareness of remembering. Curr. Direct. Psychol. Sci. 13: 49-55.

Pavlides, C. and Winson, J. 1989. Influences of hippocampal place cell firing in the awake state on the activity of these cells during subsequent sleep episodes. J. Neurosci. 9: 2907-2918.
Payne, J.D. and Nadel, L. 2004. Sleep, dreams, and memory consolidation: The role of the stress hormone cortisol. Learn. Mem. (this issue).

Ribeiro, S. and Nicolelis, M.A.L. 2004. Reverberation, storage and post-synaptic propagation of memories during sleep. Learn. Mem. (this issue).

Rock, A. 2004. The mind at night: The new science of how and why we dream. Basic Books, New York.

Roffwarg, H.P., Herman, J.H., Bowe-Anders, C., and Tauber, E.S. 1978. The effects of sustained alterations of waking visual input on dream content. In The mind in sleep (eds. A.M. Arkis et al.). Erlbaum Associates, Hillsdale, NJ.

Schacter, D.L. 1996. Searching for memory: The brain, the mind, and the past. Basic Books, New York.

Schacter, D.L. and Tulving, E. 1994. Memory systems 1994. MIT Press, Cambridge, MA.

Schwartz, S., Maquet, P., and Frith, C. 2002. Neural correlates of perceptual learning: A functional MRI study of visual texture discrimination. Proc. Natl. Acad. Sci. 99: 17137-17142.

Sederberg, P.B., Kahana, M.J., Howard, M.W., Donner, E.J., and Madsen, J.R. 2003. Theta and gamma oscillations during encoding predict subsequent recall. J. Neurosci. 23: 10809-10814.

Shimamura, A.P. 2002. Relational binding theory and the role of consolidation in memory retrieval. In Neuropsychology of memory (eds. L.R. Squire and D.L. Schacter), pp. 61-72. Guilford Press, New York.

Siegel, J.M., Manger, P.R., Nienhuis, R., Fahringer, H.M., Shalita, T., and Pettigrew, J.D. 1999. Sleep in the platypus. Neuroscience 91: 391-400.

Sirota, A., Csicsvari, J., Buhl, D., and Buzsáki, G. 2003. Communication between neocortex and hippocampus during sleep in rodents. Proc. Natl. Acad. Sci. 100: 2065-2069.

Smith, C., Nixon, M., and Nader, R. 2004. Posttraining increases in REM sleep intensity implicate REM sleep in memory processing and provide a biological marker of learning potential. Learn. Mem. (this issue).

Squire, L.R. 1987. Memory and brain. Oxford University Press, New York.

Squire, L.R. and Alvarez, P. 1995. Retrograde amnesia and memory consolidation: A neurobiological perspective. Curr. Opin. Neurobiol. 5: 169-177.

Squire, L.R. and Butters, N., eds. 1984. Neuropsychology of memory, first edition. Guilford Press, New York.

. 1992. Neuropsychology of memory, 2nd ed. Guilford Press, New York.

Squire, L.R. and Schacter, D.L., eds. 2002. Neuropsychology of memory, third edition. Guilford Press, New York.

Staba, R.J., Wilson, C.L., Fried, I., and Engel Jr., J., 2002. Single neuron burst firing in the human hippocampus during sleep. Hippocampus 12: $724-734$.

Steriade, M. 2004. Acetylcholine systems and rhythmic activities during the waking-sleep cycle. Prog. Brain Res. 145: 179-196.

Stickgold, R. 2003. Human studies of sleep and off-line memory reprocessing. In Sleep and brain plasticity (eds. P. Maquet et al.), pp. 41-63. Oxford University Press, New York.

Stickgold, R., Hobson, J.A., Fosse, R., and Fosse, M. 2001. Sleep, learning, and dreams: Off-line memory reprocessing. Science 294: 1052-1057.

Takahashi, E., Ohki, K., and Miyashita, Y. 2002. The role of the parahippocampal gyrus in source memory for external and internal events. Neuroreport 13: 1951-1956.

Varela, F.J., ed. 1997. Sleeping, dreaming, and dying: An exploration of consciousness with the Dalai Lama. Wisdom Publications, Boston, MA.

Wagner, U., Gais, S., Haider, H., Verleger, R., and Born, J. 2004. Sleep inspires insight. Nature 427: 352-355.

Walker, M.P., Brakefield, T., Hobson, J.A., and Stickgold, R. 2003. Dissociable stages of human memory consolidation and reconsolidation. Nature 425: 616-620.

Wilson, M.A. and McNaughton, B.L. 1994. Reactivation of hippocampal ensemble memories during sleep. Science 265: 676-679.

Winson, J. 1985. Brain and psyche: The biology of the unconscious. Anchor Press/Doubleday, Garden City, NY.

2002. The meaning of dreams. Scientific American Special Issue 12: $54-61$.

. 2004. To sleep, perchance to dream. Learn. Mem. (this issue).

Winson, J. and Abzug, C. 1977. Gating of neuronal transmission in the hippocampus: Efficacy of transmission varies with behavioral state. Science 196: 1223-1225. 


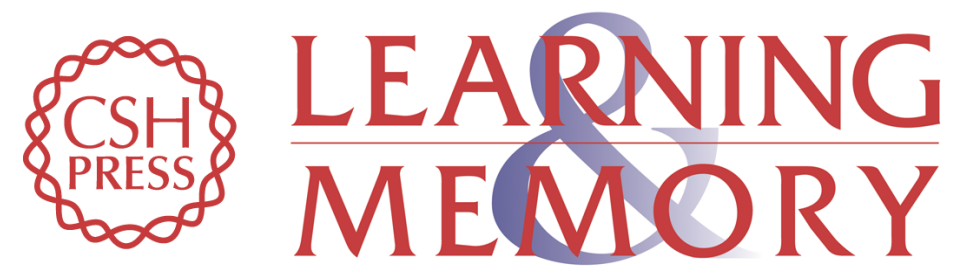

\section{Memory reactivation and consolidation during sleep}

Ken A. Paller and Joel L. Voss

Learn. Mem. 2004, 11:

Access the most recent version at doi:10.1101/lm.75704

References This article cites 37 articles, 12 of which can be accessed free at: http://learnmem.cshlp.org/content/11/6/664.full.html\#ref-list-1

License

Email Alerting Receive free email alerts when new articles cite this article - sign up in the box at the Service top right corner of the article or click here. 